\title{
tic\&société
}

Vol. 6, $\mathrm{N}^{\circ} 1$ | Second semestre 2012

Mutations du journalisme : nouveaux dispositifs, nouvelles pratiques

\section{Le défi de la construction d'un public pour le journalisme au temps de Google Analytics}

\section{François DEMERS}

\section{(2) OpenEdition \\ Journals}

Édition électronique

URL : http://journals.openedition.org/ticetsociete/1162

DOI : 10.4000/ticetsociete.1162

\section{Éditeur}

Association ARTIC

\section{Référence électronique}

François DEMERS, «Le défı de la construction d'un public pour le journalisme au temps de Google Analytics », tic\&société [En ligne], Vol. 6, N1 I Second semestre 2012, mis en ligne le 28 novembre 2012, consulté le 19 avril 2019. URL : http://journals.openedition.org/ticetsociete/1162 ; DOI : 10.4000/ticetsociete.1162 


\title{
Le défi de la construction d'un public pour le journalisme au temps de Google Analytics
}

\author{
François DEMERS \\ Faculté des Lettres \\ Université Laval \\ Pavillon Louis-Jacques-Casault \\ bureau 5413 \\ Faculté des Lettres, Université Laval \\ Québec, Canada \\ G1K 7P4 \\ francois.demers@com.ulaval.ca
}

François DEMERS, Ph.D. en science politique, est professeur titulaire au Département d'information et de communication de l'Université Laval (ville de Québec) où il enseigne depuis 1980. Auparavant, il avait été journaliste professionnel pendant 15 ans. II a créé un cours à distance totalement sur Internet portant sur le journalisme en ligne. Il est membre de l'équipe de recherche : Pratiques novatrices en communication publique (PNCP). http://www.com.ulaval.ca/personnel/professeurs/francois demers/index.php 


\title{
Le défi de la construction d'un public pour le journalisme au temps de Google Analytics ${ }^{1}$
}

\begin{abstract}
Résumé : Ce texte entend attirer l'attention sur l'une des transformations que connaît le journalisme contemporain, soit l'abandon d'une représentation du public comme formé de citoyens au profit d'un destinataire-cible qu'il s'agit de trouver. Ce changement découle de la précarité dans laquelle les médias sont plongés par le contexte d'hyperconcurrence : se construire un public et le garder est devenu un défi majeur. En réaction, les médias tentent de bénéficier des outils informatiques qui quadrillent de façon de plus en plus extensive les comportements des consommateurs sur l'Internet. Ce texte rappelle d'abord le lien traditionnel entre le journalisme, en tant que recruteur de publics, et l'industrie médiatique. Puis il porte le regard sur la place centrale que jouait la conception du public maintenant battue en brèche, dans le rapport des journalistes aux entreprises qui utilisent leur production. Enfin, il évoque les déboires et expérimentations que vivent, sur le front du recrutement et de la rétention de leurs publics, trois médias canadiens que l'auteur a eu la possibilité de fréquenter de plus près au cours des dernières années, le Reader's Digest, le Voir et le site Graffici.ca.
\end{abstract}

Mot clés : journalisme, médias, publics, destinataire, outils informatiques, Reader's Digest, Voir, Graffici.ca

Abstract: This article draws attention to one of the many transformations of contemporary journalism: the actors involved, especially professionnal journalists, increasingly replace their representation of the public as being composed of citizens by a view in which the public is a mass of targetrecipients to be found.This shift is the direct result of the instability of the media's current « hyper-competitive » environment : developing a public and keeping it is now a major challenge, for the media and for journalism. In response, the media tries to mobilize computing tools that monitor each day more intensively and extensively consumer behaviors on the Internet. This

\footnotetext{
${ }^{1}$ http://www.google.com/intl/fr/analytics/. II existe d'autres services privés de statistiques de fréquentation. Celui de Google est gratuit du moins pour son service de base et il est le plus fréquenté.
} 
Le défi de la construction d'un public pour le journalisme au temps de Google Analytics

text begins by revisiting the traditional link between journalism, as a gatherer of publics, and the media industry. It then examines the central role that the representation of the public as citizens, already undermined, plays in the relations between professionnal journalists and the media that use their production. Finally, it discusses the audience recruiting and retention efforts and disappointments of three Canadian media that the author has been close to during the last few years: Reader's Digest Canada, Voir and the website Graffici.ca.

Keywords : journalism, media, public, addressee, computing tools, Reader's Digest, Voir, Graffici.ca

Resumen: Este artículo busca llamar la atención sobre una de las tranformaciones que atraviesa el periodismo contemporáneo, cual es el abandono de la representación del público, en tanto que ciudadanos, en favor de un destinario-objetivo que debe ser buscado. Este cambio es el resultado de la precariedad en la que se sitúan los medios, como consecuencia de la hipercompetencia que les caracteriza: construirse un público y conservarlo se ha convertido en un cuestión fundamental. De esta forma, los medios de comunicación intentan beneficiarse de las herramientas informáticas que, de manera cada vez más extensiva, analizan los comportamientos de los consumidores en Internet. Se parte de la relación tradicional que existe entre el periodismo, en tanto que reclutador de públicos, y la industria mediática. Posteriormente, se focaliza sobre el lugar central que ocupaba la concepción del público, en tanto que conjunto de ciudadanos, en la relaciónn entre periodistas y empresas mediáticas; concepción que hoy se ha transformado. Por último, se evocan los problemas y experimentos en el reclutamiento y en la retención de los públicos, en tres medios de comunicación canadienses que el autor ha tenido ocasión de frecuentar y de observar en el transcurso de los últimos años: Reader's Digest, Voir y el sitio Graffici.ca.

Palabras clave: periodismo, medios, público, audiencias, destinatario, herramientas informaticos, Reader's Digest, Voir, Graffici.ca 


\title{
François DEMERS
}

\begin{abstract}
"À cette vision biopolitique (ndlr : de Michel Foucault), Gilles Deleuze ajoute un autre élément en 1990 lorsqu'il caractérise les sociétés contemporaines comme des "sociétés de contrôle". Des sociétés façonnées selon le modèle managérial du capitalisme postfordiste et s'appuyant sur l'informatique, le modèle entrepreneurial pénètre toutes les institutions. " (Mattelart, Sénécal, $2011:$ 200-201).
\end{abstract}

Le texte qui suit fait écho à cette proposition générale d'Armand Mattelart en ce qu'il éclaire quelque peu la pénétration dudit modèle entrepreneurial dans l'institution ${ }^{2}$ journalistique. II vise cependant un aspect très limité de la transformation multiforme que la "société de contrôle" impulse, soit l'augmentation de la dépendance des médias et des journalistes aux goûts et préférences de leurs clients, simples consommateurs ou annonceurs. L'informatisation généralisée des pratiques permet au management de traduire cette dépendance en une course au profilage des individus et des groupes formant publics, à toutes sortes de fins ${ }^{3}$ (Pronovost et al., 2012) et par toutes sortes de moyens (Turcotte, 2012). Dans le cas du journalisme, la participation à cette course est même devenue incontournable, une condition de survie.

L'actuel cadre général de la pratique du journalisme est celle du glissement progressif des médias traditionnels vers l'Internet pendant qu'apparaissent sur ce support d'autres diffuseurs d'information. Sur l'Internet, les médias, venus de

2 Le terme institution peut convenir au journalisme dans la mesure où il désigne un ensemble de règles, de pratiques acceptées par la société et de croyances partagées, et que sa configuration industrielle apparue dans la deuxième moitié du XXle siècle a offert une certaine permanence dans le temps.

${ }^{3}$ Le chercheur espagnol Daniel Gayo-Avello a par exemple montré qu'on peut déduire de nombreuses caractéristiques personnelles à propos d'un utilisateur du site de microblogage Twitter simplement par les données sur les personnes qu'il choisit de suivre. (Belot, 2011) 
Le défi de la construction d'un public pour le journalisme au temps de Google Analytics

l'extérieur du Net ou natifs de ce support, développent et disposent de divers outils qui leur permettent de construire des banques de données sur les internautes qui les fréquentent. Ils peuvent suivre toutes les activités de leurs clients en ligne, tester en continu la réception de nouvelles façons de faire et les adapter au fur et à mesure. Ils peuvent de minute en minute ajuster les menus des informations et les échanges discursifs qu'ils proposent. Leur course vers le Web des petits écrans (tablettes et téléphones intelligents) et de la mobilité du récepteur s'annonce plus exigeante encore en matière d'adaptation des produits au plus proche des attentes et des consommations. Ainsi va croissante l'utilisation d'outils de veille, de surveillance des publics, prenant notamment en considération la popularité des mots, des thèmes, des auteurs, des opinions, des points de vue, ou bien encore des esthétiques.

Cette dépendance accrue envers les publics, comme le rappellera la première partie du texte, est une conséquence directe du régime d'hyperconcurrence que l'économie de l'innovation promeut et célèbre. L'entrée du journalisme dans ce nouveau monde, qui soutiendra la deuxième partie du texte, était conditionnelle à l'élimination du blocage organisationnel et idéologique que formait, dans le discours dominant à propos du journalisme, la conception du public comme ensemble de citoyens éclairés ou cherchant à l'être. À ce propos, l'invocation du bien commun par les journalistes est mise en exergue dans l'éclairage des propos de Pierre Bourdieu, quand il parle de l'idéal du service public attaché au travail pour l'État. Cette citation, par exemple, est ici appliquée aux journalistes: "Ceux qui, comme Marx, inversent l'image officielle que la bureaucratie entend donner d'elle-même et décrivent les bureaucrates comme des usurpateurs de l'universel, agissant en propriétaires privés des ressources publiques, ignorent les effets bien réels de la référence obligée aux valeurs de neutralité et de dévouement désintéressé au bien public qui s'impose avec une force croissante aux fonctionnaires d'État à mesure qu'avance l'histoire du long travail de construction symbolique au terme duquel s'invente et s'impose la représentation officielle de l'État comme lieu de l'universalité et du service de l'intérêt général. » (Bourdieu, 1994 : 131) L'idéal du service public peut en effet être considéré comme un pilier de l'illusio des journalistes par analogie avec les bureaucrates (Costey, 2009). II est une condition de la réalisation de l'action et, en même temps, il cache d'autres dimensions de cette action que l'observation peut dévoiler.

La troisième partie du texte présente trois cas de médias forcés d'affronter de manière prioritaire le défi de la construction d'un public stable, médias que nous avons été amené à suivre de plus près. Elle met en scène la réaction de la firme Reader's Digest Canada au changement général de contexte de l'industrie médiatique et des magazines plus particulièrement, les initiatives récentes de la 


\section{François DEMERS}

chaîne d'hebdomadaires culturels Voir, ainsi que l'aventure du site journalistique Graffici.ca lancé en septembre 2011 en Gaspésie. Elle illustre l'omniprésence de la recherche des données sur les usagers dans des médias pourtant différents sous la plupart de leurs aspects.

\section{Médias et journalisme}

Au moins à partir de l'industrialisation de la presse au $\mathrm{XIX}^{\mathrm{e}}$ siècle, le journalisme et les médias ont paru tellement imbriqués l'un dans l'autre qu'il était coutumier, même chez les chercheurs, de considérer les deux termes comme désignant la même chose et de les utiliser comme des équivalents (Gagné, 1980). Dans le langage courant, on rencontre d'ailleurs encore fréquemment cet amalgame. Pourtant, de plus en plus clairement, le journalisme s'est révélé n'être qu'un des contenus des médias. Les autres contenus : publicité, fiction et autres divertissements ont peu à peu augmenté leur espace (dans la presse écrite) et leur temps d'antenne (à la radio et à la télévision où l'information n'a jamais dominé). Ce qui importe davantage dans le cadre de ce travail, c'est que l'identification des médias au journalisme traduisait aussi à l'origine une situation où les contenus journalistiques servaient de principale locomotive à la construction des publics. On lisait un journal pour les nouvelles et ses opinions. Puis, dans le cadre de la presse électronique (radio et télévision), le journalisme est devenu la portion congrue des contenus et a été secondarisé sinon marginalisé dans la capacité du média à rassembler un public.

Cette marginalisation s'est accélérée avec l'Internet, où les pages et les sites d'information journalistique sont des lieux d'attraction limités par comparaison aux autres activités (innombrables) qui sont offertes : jeux, pornographie, autopublications, conversations diverses (médias sociaux), etc. C'était déjà le cas dans les médias d'information traditionnels, mais le nouvel environnement qu'offrent les médias sur Internet augmente la difficulté pour le journalisme de se distinguer des autres activités et produits. On n'a qu'à penser au site YouTube maintenant considéré comme un média d'information (Agence FrancePresse, 2012).

Ce glissement du journalisme, à l'intérieur de la plupart des médias qui le portent, d'une position centrale vers une position de contenu 
Le défi de la construction d'un public pour le journalisme au temps de Google Analytics

d'accompagnement, sinon carrément facultatif ${ }^{4}$, est l'un des traits du déploiement des médias dans le temps. Elle a de nombreux effets dont la mort de plusieurs médias traditionnels, axés sur les nouvelles et reportages, et des perturbations majeures du marché du travail en journalisme : pertes massives d'emplois dans les médias traditionnels et création d'emplois chez les agrégateurs et dans l'information locale et micro-locale.

Tout s'est passé comme si le secteur d'activité économique des médias avait évolué selon trois axes de transformation allant dans la même direction. Comme indiqué ci-dessus, le premier se présente comme une avancée de la spécialisation, vecteur du progrès des sociétés selon Durkeim : le journalisme s'est affirmé, se distinguant de plus en plus de la publicité et des autres discours publics. Le second facteur, qui se marie au premier, repose sur l'enrichissement général dans les sociétés dites avancées : les produits, services et activités, de plus en plus nombreux et diversifiés, se disputent le budget temps et le budget tout court des publics possibles. Le troisième, macro-économique, prolonge et fait rebondir l'orientation économique de supply-side qui s'est imposé au temps de Ronald Reagan et de Margaret Thatcher. II mise sur l'innovation et l'abaissement des coûts de production pour stimuler la croissance plutôt que sur la capacité d'achat des consommateurs. Les technologies liées à la numérisation, dont l'Internet est exemplaire en matière de communications, sont le cœur de l'économie de l'innovation: le renouvellement constant des équipements, logiciels et activités proposées, y est la règle du jeu. Au tournant du siècle, la conjugaison de ces trois orientations du développement a fait apparaître "l'hyperconcurrence " (Brin et al., 2044) entre les offres, les contenus et les équipements.

Résultat global : fragmentation des marchés et volatilité des publics. Attirer et retenir un public est devenu l'obsession des médias et la condition de leur succès autant que de leur survie, au point que le passé et ses formules gagnantes ne sont plus garants de l'avenir. Chaque média doit réinventer (innover) son modèle d'affaires, sur la base de ses acquis historiques, quand il en a. Le premier grand trait de ce journalisme renouvelé vient avec la fragmentation des publics, laquelle induit la spécialisation graduelle des médias et, par conséquent, l'autonomisation des thèmes et des domaines de couverture. La nouveauté ne repose pas sur le fait qu'il y ait un journalisme "sportif ", un journalisme "économique », un journalisme "politique », et

\footnotetext{
${ }^{4}$ La faillite de la chaîne de télévision québécoise TQS en 2007, son rachat par des distributeurs de films (Remstar), et sa transformation l'année suivante en chaîne généraliste sans production d'information journalistique autonome aura été une illustration de la fragilité de l'information journalistique dans le contexte actuel.
} 


\section{François DEMERS}

d'autres rubriques du genre. Ce qui est nouveau, c'est que les spécialités - et on en invente chaque jour - ne se retrouvent plus dans les mêmes médias de masse, mais disposent de leurs propres plateformes médiatiques. Ces journalismes «spécialisés » peuvent maintenant s'inventer en tant qu'activités affranchies d'un journalisme général ou transversal. Que ce soit dans les relations aux sources, dans les techniques documentaires, dans l'écriture, et même dans l'éthique, la soumission à des normes générales n'est plus considérée comme indispensable, par les entrepreneurs, mais aussi de plus en plus souvent par les journalistes eux-mêmes. Ce qui devient la règle suprême, c'est l'adéquation des pratiques de production avec les publics visés ou les publics déjà recrutés. D'où l'exigence et l'urgence de déployer une panoplie d'instruments pour connaître et profiler ces publics.

D'autres facteurs de changement, notamment du côté du rôle social du journalisme, rendent encore plus aigu le défi de la construction d'un public pour les médias et pour le journalisme. Jean-François Tétu (2008) avait résumé les trois apports historiques du journalisme en le décrivant comme un auxiliaire.

Premièrement, dans le passage de la société traditionnelle structurée par le temps liturgique vers le temps mécanique, neutre et mesuré en parties égales. En journalisme, ce temps mathématique a imposé la temporalité du présent, nommée actualité : annuelle, mensuelle, hebdomadaire, quotidienne, hebdomadaire et maintenant en temps réel, minute par minute. La représentation de la temporalité sous une forme mécanique est devenue, par le colonialisme, un acquis et une dominante dans toutes les sociétés. L'actualité est aujourd'hui un repère universel.

Le second rôle historique aura été sa contribution à la construction du territoire national (régional et local), non seulement en épousant les réseaux de transports et de communications pour définir les aires de distribution du journal, mais aussi en assurant la transmission des discours des dirigeants, des élus et des fonctionnaires sur la base du découpage du territoire opéré par l'État, et en assurant la construction de la «communauté imaginée »(Anderson, 1995). Le journalisme va assurer la transmission verticale des messages de l'État en direction de ses sujets et de plus en plus les messages de toutes les autres formes d'élite qui quadrillent et vivent du territoire.

Enfin, troisièmement, le déploiement du journalisme est intimement lié aussi à la mise en place de la démocratie formelle avec son système électoral. Les médias offriront leurs services et leur journalisme pour assurer l'expression publique des citoyens, quand ils exercent leur raison privée dans l'espace public à propos des enjeux collectifs et des performances de l'État (Habermas, 1968). Puis, la globalisation des télécommunications et le déplacement des pouvoirs 
Le défi de la construction d'un public pour le journalisme au temps de Google Analytics

étatiques nationaux vers des lieux «mondiaux » non démocratiques ont rendu les frontières territoriales poreuses, les faisant reculer vers un découpage linguistique et une forme "virtuelle » et transnationale. Résultat, l'espace public (national), et les médias qui vont avec, sont en crise (Moffette, 2009 ; Robitaille, 2009). On constate qu'à ce jeu, la construction de publics de l'anglophonie a été favorisée d'autant plus que les médias, vecteurs de la transnationalisation, les télés d'information en continu et l'Internet ont été soutenus et promus vigoureusement par les États-Unis. Un espace transnational s'est construit sur une base linguistique dans l'univers virtuel des télécommunications.

De plus, l'information dite internationale a pris le pas sur les thèmes nationaux. Comme le souligne le politologue Jean-Herman Guay dans son bilan de la première décennie du siècle: "Les deux dernières années ont été marquées par une omniprésence des enjeux internationaux, au détriment des enjeux canadiens et québécois : le 11 septembre 2001, bien évidemment, mais aussi la guerre en Afghanistan, celle en Irak et les élections présidentielles américaines de 2000, celles de 2004 et, surtout celles de 2008 ont détourné l'attention de tout le monde, y compris au Québec. Le phénomène dépasse la conjoncture, puisqu'il se combine à la récurrence des sommets multilatéraux et des interdépendances économiques. Le monde est continuellement sur nos écrans!" (Guay, 2009). Cela se poursuit, avec le printemps arabe, la crise syrienne, la guerre avec l'Iran...

Sous un autre angle, au-delà de la bataille pour l'accès aux médias qui a marqué l'après-guerre et conduit aux "relations publiques généralisées » (Miège, 1995), la victoire morale de la démocratie sur le communisme, l'ascension subséquente de l'idée démocratique au statut d'horizon de la pensée en même temps que l'apprivoisement général de la consommation médiatique ont fait de l'expression en public de chacun, et la visibilité qui va avec (Thompson, 1995), un bien à démocratiser, augmentant la demande pour l'extension et la multiplication des moyens de communication.

En bref, ces transformations ont, elles aussi, conduit au fractionnement des publics des médias et du journalisme, après plus d'un siècle d'évolution vers des monopoles territoriaux, jusqu'aux années 1980. Elles ont aussi ouvert la voie à l'apparition de publics transnationaux en un mouvement qui positionne autrement le défi de la construction d'un public pour les anciens comme pour les nouveaux médias. D'une part, les anciens publics, reposant sur les communautés territoriales, se subdivisent et leur division pousse au regroupement des entreprises médiatiques qui cherchent à reconstruire les publics larges antérieurs par l'addition de publics de niche. Convergences des médias et de leur propriété sont à l'ordre du jour, entraînant notamment 


\section{François DEMERS}

«malaise et détresse dans la profession» journalistique (Bernier, 2008). Au Canada, l'extension des grands groupes de médias est très avancée et soutenue par les gouvernements (Baillargeon, 2012d).

Par contre, les médias qui sont en position de rejoindre des publics extérieurs à leurs frontières territoriales et linguistiques peuvent espérer étendre leur public dans cette direction. C'est notamment le cas des médias de l'élite (The New York Times, Le Monde, The Globe and Mail, etc.) qui offrent traditionnellement aux élites étrangères des informations d'initiés sur la vie des élites nationales, formant ainsi l'épine dorsale informationnelle d'un réseau transnational (Merrill, 1968). C'est aussi le cas de «nouveaux médias ", tels les agrégateurs (Google, Yahoo, etc.) ou de médias nés-sur-le-Net comme le Huffington Post, qui implantent des franchises sur plusieurs "marchés" nationaux.

\section{Publics imaginés ${ }^{5}$}

Le nouveau contexte renouvelle donc pour chaque média la question de l'identification et de la rétention d'un public. Pour les médias et les diverses "sources" préoccupées de rendre publics leurs points de vue, l'hyperconcurrence entre les messages et les producteurs de produits a rendu prioritaire la connaissance de ce que font les récepteurs de ces messages. II a aussi, par effet d'entraînement, renouvelé les priorités dans le champ scientifique qui étudie les médias, poussant les chercheurs auparavant concentrés sur les questions de la production médiatique vers les études dites de réception (Demers, 2003). Ces recherches, qui procèdent principalement par l'observation des comportements et l'analyse des discours des récepteurs ne donnent pas accès directement aux mécanismes qui rassemblent, de manière temporaire ou de façon plus stable, des groupes de clients. Mais, en couplant leurs résultats avec ceux que fournissent les profilages sociodémographiques et comportementaux que pratiquent les services commerciaux des médias, il est possible, du moins l'espère-t-on, de prévoir plus précisément les résultats des expérimentations auxquelles se livrent les entreprises dans leur recherche de marchés.

\footnotetext{
${ }^{5}$ Aux yeux des chercheurs, le public des médias reste avant et après Internet, « une entité invisible, une simple création discursive, une construction intellectuelle qui n'est jamais observable en tant que telle. » (Rieffel, 2005, p. 183)
} 
A priori, sur le terrain commercial, les médias traditionnels ont une longueur d'avance. Ils peuvent compter sur divers mécanismes de " connaissance » des usagers, actuels et potentiels, méthodes quantitatives et qualitatives qui ont été rodées au fil du temps dans leurs propres murs ou par des consultants: les cotes d'écoute, les sondages, les focus group, l'interprétation des lettres de lecteurs, des téléphones, les enquêtes par échantillons et par panels, etc. À cela, Internet a ajouté toutes sortes d'activités interactives avec des interlocuteurs abonnés, de passage, curieux, anonymes, etc. Sans compter, l'accès plus facile aux produits des concurrents ainsi que l'analyse de leurs innovations et de leurs "meilleures pratiques". Les médias venus du passé bénéficient aussi d'une vaste expérience de cet autre versant de la "science " du marketing : les moyens de « forcer " l'attention et la fascination des cibles. Ils peuvent crier plus fort que les autres par leur publicité. Ils peuvent se positionner avantageusement en titillant des «besoins" inavoués ou inavouables de clients potentiels. Ils peuvent faciliter la consommation par une distribution plus rapide, par des primes à l'abonnement, des services ajoutés, etc.

Longtemps, les journalistes, du moins ceux dits de la presse de qualité, ont été à l'abri de ces préoccupations et opérations. Plus exactement, dans la vie quotidienne, la question du public était pour eux un point aveugle. Pour trois raisons. D'abord, parce que le discours de justification publique de la raison d'être du journalisme était entièrement - il l'est encore largement - ancré dans l'éthos du service public: le journalisme est là pour soutenir l'expression publique des opinions et le dévoilement des données factuelles indispensables au citoyen pour comprendre l'action de l'État et les projets des politiciens en matière de bien commun. Dans l'imaginaire des journalistes, dans les discours de leurs représentants et dans celui de la majorité des observateurs savants, le citoyen comme destinataire du discours journalistique est connu et fixe : c'est une personne rationnelle et préoccupée de comprendre la société et sa vie politique. C'est la représentation du citoyen mise de l'avant par Les Lumières et la Modernité, celle qui est à la base de la conception actuelle de la démocratie comme arène de "communautés conflictuelles " où se discutent les enjeux publics (Muhlmann, 2004). Le journaliste donne à ce citoyen imaginé (son destinataire) le matériel dont il a besoin pour agir en tant que membre actif de sa communauté. Cette conception justifie aussi que le journaliste soit un intervenant et un éducateur en ce qu'il doit élever son public vers une citoyenneté éclairée et active. II devrait prolonger l'éducation au besoin.

Bien sûr, en pratique, dans la "formation discursive" du journalisme (Ringoot et Utard, 2005), cet imaginaire a donné naissance à au moins deux discours. Celui, noble, qui invoque les besoins du citoyen politique en matière 


\section{François DEMERS}

d'information, sérieuse, vérifiée et centrée sur le bien public. Celui, honteux (hors des salles de rédaction), qui pointe les lacunes du public réel assoiffé « de sport, de sexe et de sang " afin de justifier des choix éditoriaux qui fournissent «ce que le public veut savoir plutôt que ce qu'il doit savoir ».

La référence à la conception - dominante - d'un public universel permettait aussi aux journalistes de revendiquer une compétence indépendante de l'entreprise employeur. C'était l'un des piliers de leur "profession » en tant qu'expertise transversale pouvant être utilisée dans les différents contextes médiatiques. Cette conception a commencé à être délégitimée très tôt (Demers, 1989) ; elle se révélait incompatible avec le mode de gestion postfordiste fondé sur l'intéressement - financier, sinon émotionnel - qui a commencé à se mettre en place dans les années 1980, y compris dans les médias. Si bien que l'exdirecteur général de l'information à Radio-Canada, Alain Saulnier, se voit maintenant forcé de conclure : «... un journaliste peut difficilement aujourd'hui se définir comme journaliste d'abord, puis comme employé de tel ou tel média ». (2012).

Compte tenu de la prégnance de cet imaginaire dans le milieu journalistique et chez les élites jusqu'à tard dans le $X X^{e}$ siècle, les médias en tant qu'entreprises ont développé une procédure de gestion confiant la question du public à d'autres services que la rédaction : à la distribution, à la promotion ou aux services commerciaux. C'est de ce côté de la machine administrative que se sont développées la préoccupation et l'expertise d'écoute et d'interrogation des publics. L'isolement de la Rédaction sur cette question n'était cependant pas complet, car les cadres supérieurs de l'entreprise et du Service de la rédaction assuraient la traduction et la percolation des données recueillies par les autres services en direction des journalistes. Pour cela, ils devaient inventer une façon euphémisée - c'est là la troisième raison du point aveugle dans la doxa journalistique -, qui pouvait rendre compatible le portrait du public, dont ils avaient le secret, et notamment ses réactions au produit journalistique offert, avec le destinataire mis de l'avant par la doxa journalistique.

Au fil du temps, les cadres ont réussi à mettre en forme des façons de dire qui justifient aux yeux des journalistes, l'importance accordée aux sports professionnels, aux faits divers sanglants, aux potins sur les gens riches et célèbres, à la starification des élites et la vedettisation de la politique. Souvent, sous forme de concessions discursives stratégiques - c'est triste, mais inévitable -, ou d'invocations tordues de l'idéal démocratique - par exemple " l'attrait du public pour ces contenus peut aussi être considéré comme un phénomène public socialement important ... et donc légitime ». 
Le défi de la construction d'un public pour le journalisme au temps de Google Analytics

Le mécanisme de séparation des domaines entre les sections administratives de l'entreprise, définies comme des caissons étanches communiquant uniquement par en haut, était aussi celui qui gouvernait les rapports entre les annonces et les contenus d'information journalistique. Dans les grandes salles de rédaction, les espaces d'annonces du journal quotidien du lendemain ou du bulletin de nouvelles étaient attribués par le sommet des hiérarchies, de telle sorte que l'espace d'information se construisait autour de ces espaces de publicité sans savoir ce qu'il y aurait précisément dans l'espace publicitaire à côté. Façon de diminuer les risques de conflits entre les messages placés côte à côte, par exemple la promotion d'un produit ou d'une marque qui, dans l'espace d'information à côté, ferait l'objet d'une dénonciation. Refus aussi des journalistes d'être de quelque façon influencés par leur connaissance préalable des messages des commanditaires.

Puis, la digue s'est rompue quelque part au tournant du siècle. La nouvelle précarité des médias a forcé la prise en compte de plus en plus fine par les journalistes des « désirs » et comportements des publics. Le courant de pensée nord-américain inspiré de la grass-roots democracy a vu dans les possibilités d'expression publique offerte par l'Internet, une façon de contourner le passage obligé par les grands médias et leurs journalistes. Le journalisme citoyen est né. Les dogmes journalistiques ont craqué les uns après les autres. Pour les reporters, l'idéologie professionnelle de l'objectivité a perdu sa légitimité au profit du «tout est affaire d'opinion ». Le bien commun, qui devait guider les choix éditoriaux, est désormais une chimère puisque tout est affaire de coalitions circonstancielles et de majorités d'opinions, temporaires et passagères. L'indépendance professionnelle des journalistes est un leurre, diton maintenant volontiers, puisqu'ils sont entièrement des participants / parties prenantes, enthousiastes de préférence, de l'entreprise qui les emploie. L'idée d'un citoyen rationnel qu'il s'agirait d'éclairer est d'un autre temps : le citoyen d'aujourd'hui est ce qu'il est et il n'y a pas de raison pour que l'opinion éditoriale d'un journaliste ait plus de valeur ou priorité. Les études d'audience ont d'ailleurs montré sa résistance aux tentatives d'éducation. Quant aux cultural studies, elles ont clairement établi que le journalisme de papa était mâle, blanc, et occidental (Hall, 2007).

Pour résumer, le balancier a basculé du côté du consommateur... du moins dans la façon dominante de percevoir et de raisonner. Pour les journalistes, il faut d'abord sauver l'entreprise - son gagne-pain — en contribuant, autant que faire se peut, à la construction d'un groupe de consommateurs réguliers. Le changement de la perspective dominante des journalistes en direction d'une subordination aux "goûts » du public a été, et est soutenu, accompagné, provoqué au besoin par le changement symétrique de perspective dans le 


\section{François DEMERS}

champ plus large de la gestion et des relations industrielles qui se sont mises en place dans les années 1970 / 1980 et que l'on nomme le post-fordisme. II importe donc que tous les participants à l'entreprise - les stake-holders - , pas uniquement les ingénieurs et autres cadres, se montrent intéressés à son succès, et même mobilisés émotionnellement en ce sens, jusqu'à la façon Walmart / McDonald's.

\section{Le panopticon parfait}

Mais, pour plaire au public, pour deviner ses désirs et ses goûts, il faut l'étudier. Or, en principe, l'Internet, support universel pour tous les médias et les processus de communication, pourrait offrir à l'observateur une vue panoramique complète de toutes les activités qui s'y déroulent. II offre le mirage du panopticon parfait: l'observateur a en main les outils informatiques pour suivre dans le détail, ce qui se déroule dans tous les recoins et même suivre à la trace les comportements de tel ou tel participant. Les chercheurs sont fascinés par ces possibilités: disposer d'un terrain totalement quadrillable. II deviendra analysable exhaustivement à l'aide de robots et de méthodes quantitatives (Rebillard, 2011). L'Internet forme un champ clos autorisant une observation absolue. A priori donc, les médias présents sur le site peuvent récolter sans limites les données comportementales des usagers. Ils peuvent les regrouper de façon à faire ressortir des profils et des majorités. Ils peuvent aussi croiser ces données avec celles que fournissent les statistiques gouvernementales sur les populations. L'actuel mouvement de pression pour l'ouverture des données étatiques aux entrepreneurs privés va en ce sens. Ce mouvement est l'incarnation la plus récente des revendications pour l'accès à l'information gouvernementale et l'exigence démocratique de transparence de l'État qui a pris son envol dans les années 1980 et que l'on exprime aujourd'hui en termes de e-gouvernement. Ces deux groupes de données peuvent également être croisées avec d'autres données recueillies par d'autres entreprises dans le cadre de leurs opérations de marketing. II existe d'ailleurs un marché de vente et d'achat de ce type de données depuis très longtemps. On n'a qu'à penser aux profils de solvabilité du client qui sont accessibles aux marchands par les services payants de firmes spécialisées.

Cela n'est pas vraiment neuf, ce qui l'est pour le journalisme, c'est que ces données soient désormais systématiquement l'objet de discussion dans les salles de rédaction. Le thème du public n'y est plus tabou, il fait partie des conversations et est couramment invoqué en vue de prises de décision. Du moins, nous avons pu le constater au cours des dernières années à partir de 
Le défi de la construction d'un public pour le journalisme au temps de Google Analytics

l'accès à trois entreprises de presse : Reader's Digest Canada, l'hebdomadaire Voir et le site Graffici.ca.

En ce qui a trait au Reader's Digest, l'accès à des documents confidentiels au cours des dernières années et des conversations avec le rédacteur en chef Robert Goyette ont permis de percevoir l'ampleur des secousses que subit le magazine canadien au plus fort tirage ${ }^{6}$, membre d'une famille internationale de succursales. Par cette fenêtre, il a aussi été possible de découvrir le mur que frappe l'industrie des magazines. Les reculs de tirages et de revenus sont énormes, pour l'ensemble du secteur: de l'ordre du tiers entre 2003 et 2010 dans la francophonie canadienne (Giroux, 2012). En 2011, pour le Sélection du Reader's Digest, c'est près de $10 \%$ (Baillargeon, 2012e). Pour l'édition anglaise, le recul est encore plus prononcé. $R D$ est une entreprise d'expérience, il semble pourtant que la transformation du marché de la publicité, avec glissement vers le Net et effondrement des tarifs publicitaires sous l'action des grands agrégateurs, lui donne de forts coups de butoir. De plus, la distribution du magazine papier essentiellement par le service postal est victime d'une augmentation des coûts et, là encore, de la transformation du système postal canadien dont les routes les plus rentables sont dépecées au profit d'entreprises privées tandis qu'une bonne partie des contenus est désormais acheminée par voie électronique. Enfin, la concurrence de l'Internet, où l'offre de jeux éducatifs, inoffensifs ou casinos - prolifère, ébranle l'une des pratiques gagnantes de l'entreprise consistant à fidéliser une bonne partie de ses consommateurs à l'aide de diverses formules de loteries. Reader's Digest, misant sur ses habiletés en matière d'analyse de listes d'abonnés et de données diverses sur les clientèles, fait présentement l'apprentissage à la dure des possibilités de traduction sur le Net de son expérience en ces matières, en terme de contenus, mais aussi et surtout en termes d'attrait et rétention des publics. Car elle travaille systématiquement son passage sur le $\mathrm{Web}^{7}$ en même temps qu'elle renouvelle ses versions sur papier et augmente même le nombre de ses titres (Our Canada, More of Our Canada, Best Health). Ses employés

\footnotetext{
${ }^{6}$ II y a 2 ans, selon le rapport du Print Measurement Bureau (PMB), le Reader's Digest demeurait la publication canadienne la plus lue, soit 6,3 millions de lecteurs par numéro (Baillargeon, avril 2010). Le mois précédent, une enquête de la Columbia Journalism Review (CJR) auprès de 665 éditeurs de magazines américains établissait que «les magazines ne font pas de profit sur Internet » et qu' "il n'y a pas de modèle clair pour gagner de l'argent ». (Cauchon, 2010)

${ }^{7}$ http://www.readersdigest.ca/ et http://selection.readersdigest.ca/magazine/. Voir aussi à travers un moteur de recherche, le déploiement de la présence du Reader's Digest par le truchement de plusieurs portes d'entrée et sites. Voir aussi le degré de sophistication de l'interactivité, des animations et des vidéos.
} 


\section{François DEMERS}

sont très identifiés à l'entreprise et sont alimentés constamment de statistiques sur les performances des titres, des thèmes, des angles, etc.

Pour ce qui est de l'hebdomadaire Voir, les observations ont été faites grâce à des rencontres avec certains de ses artisans qui sont d'anciens étudiants de I'Université Laval. Voir est un success-story des années $1980^{\circ}$, une presse gratuite format tabloïd, spécialisée dans l'information dite culturelle. Au Québec, le groupe Voir a été le premier à développer une présence sur le Net centrée sur diverses formes d'interactivité avec les lecteurs. L'important, pour les fins de ce texte, c'est le fait que Voir, à l'occasion de la crise économique de 20082009, a touché un plafond. Ses dirigeants ont alors découvert l'ampleur de l'érosion cumulative des tarifs publicitaires (Baillargeon, 2012c) pour tout ce qui peut s'annoncer sur les sites agrégateurs et moteurs de recherche couvrant de larges zones linguistiques - en Amérique du Nord, l'anglais - ou ayant les moyens de développer leurs services en plusieurs langues. Les tarifs seraient passés en cinq ans de quelque $30 \$$ par mille pages vues (CPM) à $1 \$$ et moins. L'impact de la concentration des revenus publicitaires entre les mains de quelques grands opérateurs états-uniens est en effet considérable. "Already in 2011, five technology companies accounted for $68 \%$ of all online ad revenue, and that list does not include Amazon and Apple, which get most of their dollars from transactions, downloads and devices. By 2015, Facebook is expected to account for one out of every five digital display ads sold. " (StateoftheMedia, 2012) Au Canada, Voir n'est pas la seule victime de l'effondrement des tarifs publicitaires comme l'indiquent les récentes fermetures de sites par le groupe Rogers, pourtant l'un des plus grands groupes médias canadiens, notamment en câblodistribution et dans le téléphone sans fil (Baillargeon, 2012b).

Voir a aussi été secoué par l'offensive du Huffington Post (Baillargeon, 2012f) qui a lancé une édition québécoise en français ${ }^{9}$, après avoir implanté une succursale au Canada anglophone ${ }^{10}$. Voir a aussitôt lancé une contre-offensive sur le terrain qui fait la force de la formule HuffPost, laquelle s'appuie en grande partie sur des blogueurs volontaires, une pratique que Voir a lui aussi développée. Au lendemain de l'annonce de l'arrivée du Huffington Post, Voir a lancé une campagne d'opinion pour décourager diverses personnalités de se prêter au jeu d'un concurrent étranger qui viendrait faire de l'argent en exploitant du travail gratuit. II a aussi et surtout annoncé une entente avec une longue liste de blogueurs qu'il rémunérera désormais (Baillargeon, $2012 \mathrm{~g}$ ). On ne sait pas

\footnotetext{
${ }^{8}$ Le premier numéro est sorti des presses en novembre 1986.

${ }^{9}$ http://quebec.huffingtonpost.ca/

${ }^{10} \mathrm{http}: / /$ www.huffingtonpost.ca/
} 
Le défi de la construction d'un public pour le journalisme au temps de Google Analytics

encore si l'implantation du HuffPost est une réussite, ni dans quelle mesure le Voir a réussi à stopper l'hémorragie potentielle de blogueurs-citoyens au profit de son concurrent américain.

En résumé, Voir tente depuis la crise de 2008 d'opérer un repli et un redéploiement dans l'ordre, en fermant des succursales (Baillargeon, 2012a), en coupant ici et là (Allard, 2012) tout en ajoutant des services. Par exemple, il s'est emparé de la gestion des présentoirs publics pour les publications gratuites. Il est aussi devenu producteur de contenus télévisuels (Voir sur TéléQuébec et Guide Restos Voir sur Évasion; etc.). Par ailleurs, il met actuellement à l'épreuve de nouvelles façons de fidéliser à la fois des annonceurs et des consommateurs en les couplant par le biais d'un système de cartes de crédit dédiées. ${ }^{11}$ L'opération suppose une exploitation ciblée et délicate des «profils » de lecteurs et de cyber-usagers du Voir. La formule est actuellement à l'essai principalement dans le commerce de la restauration.

Troisième cas, celui du site d'information Graffici.ca ${ }^{12}$ qui a été lancé en septembre 2011 en Gaspésie, région maritime du sud-est du Québec notamment connue dans l'industrie touristique pour son Rocher Percé (Demers, 2012). Nous nous sommes associé à cette aventure depuis fin 2010 dans le cadre d'un projet de «laboratoire vivant ${ }^{13}$ soutenu par l'organisme CEFRIO ${ }^{14}$.

En fait, le projet d'un site gaspésien d'information a pris naissance en 2010 dans les bureaux du magazine mensuel Graffici apparu en 2001 comme journal

${ }^{11}$ http://boutique.voir.ca/fonctionnement/.

12 http://www.graffici.ca/

${ }^{13}$ Pour le CEFRIO, il s'agit de tester la pertinence de la formule européenne nommée " living lab» en matière d'innovation sociale, avec souci de promotion de l'usage des nouvelles technologies. Le CEFRIO a choisi d'expérimenter à travers deux projets, dont celui du Graffici.ca, la mise en place de laboratoires où sont appelés à collaborer systématiquement des chercheurs académiques et des acteurs des milieux de pratique. < http://www.openlivinglabs.eu/> Dans le projet Graffici.ca, le CEFRIO jouant le rôle d'animateur, des chercheurs des Universités Laval et de l'Université du Québec à Rimouski (UQAR), des étudiants ainsi que des experts médias issus de l'entreprise privée sont mis en interaction avec les artisans du journal Graffici, porteurs du projet d'un site/portail d'information journalistique.

${ }^{14}$ Le Centre de francisation des technologies de l'information dans les organisations (CEFRIO) joue depuis 25 ans en 2012 un rôle d'intégration des TIC à la société québécoise, Au tout début, il s'agissait, entre autres, de s'assurer de la francisation des outils et des équipements lors de l'informatisation. Alors que maintenant, il s'agit d'intégrer les TIC dans les façons de faire afin d'améliorer les services à la société et la compétitivité des entreprises tant privées que publiques. <http://www.cefrio.qc.ca/> 


\section{François DEMERS}

gratuit spécialisé dans l'information culturelle - comme Voir. Coopérative de solidarité, il fait partie de l'association des journaux communautaires. En 2006, il s'est transformé en mensuel d'informations générales et a atteint la rentabilité. En effet, 5 ans plus tard, il publiait 30 à 40 pages par numéro, comptait sur un budget annuel d'environ $800000 \$$, distribuait 40000 copies, rejoignait 60 à $70 \%$ des lecteurs potentiels - entre 60000 et 65000 - en étant distribué dans tous les foyers de la Gaspésie, donnait du travail à 10 employés réguliers, 15 pigistes, 50 collaborateurs, et occupait 2 bureaux (New-Richmond et Gaspé).

Des consultations diverses ont conduit à un consensus sur un déficit communicationnel : le territoire est pourtant quadrillé par de nombreux médias ${ }^{15}$, plusieurs depuis l'extérieur il est vrai, et un grand nombre à portée locale ou sous-régionale seulement. Aucun ne le fait dans une perspective de développement d'une conscience collective pour l'ensemble du territoire. Mais c'est le réseau de sympathisants construit par les artisans de Graffici papier dans les milieux de l'animation, sociale, culturelle, économique et même politique, du côté de la Conférence régionale des élus (CRÉ) notamment, qui a permis de penser que la mise en place d'un site d'information couvrant l'ensemble de la Gaspésie ${ }^{16}$ était sérieusement envisageable, malgré l'énormité du défi. En effet, la Gaspésie couvre un territoire très étendu où les communications (routes, trains, avions) sont un défi constant en raison des distances. Cet immense territoire est aussi peu peuplé : moins de 100000 habitants adultes tandis que le tissu économique est très dépendant des ressources naturelles - bois, mines, pêcheries surtout - secteurs fragilisés par la crise. Finalement, les promoteurs ont réussi à ramasser quelque 800000 \$ pour lancer le site et le soutenir jusqu'en mars 2013. Le site a été lancé au début de septembre 2011. Son objectif d'achalandage était de 40000 visiteurs uniques après 6 mois. II en comptait 48000 après 5 mois.

Dans le cadre de ce laboratoire vivant, nous avons participé à diverses opérations dont la mise en place de blogueurs citoyens dès le lancement et la " couverture spéciale » à l'aide de téléphones intelligents lors de l'Université Rurale $2011^{17}$ dans la première semaine d'opération du site. Au printemps 2012 , nous avons procédé avec un consultant externe à un audit en 12 points du site

\footnotetext{
${ }^{15}$ Voir : http://www.gaspesieilesdelamadeleine.gouv.qc.ca/medias/RepertoireMedia.asp? region=Gaspe

${ }^{16}$ Six municipalités régionales de comté (MRC) si on inclut les îles-de-la-Madeleine, représentées par le sigle GIM.

17 Un colloque d'une semaine organisé par l'Université du Québec à Rimouski. [http://www.urq2011.com/]
} 
Le défi de la construction d'un public pour le journalisme au temps de Google Analytics

- graphisme, esthétique, ergonomie, navigation, traitement journalistique - et à un examen des données de Google Analytics pour les opérations de mai 2012.

Voici un aperçu de quelques constats généraux tirés de Google Analytics :

- $57,74 \%$ des visites proviennent de sites référents (principalement Facebook) ; 27,42\% sont issues du trafic de recherche (moteurs de recherche) ; 14,84\% constituent des accès directs sur le site.

- Le mot Graffici se situe bon premier (20\%) dans la recherche par mot-clé. C'est donc dire que ceux qui l'ont utilisé voulaient clairement aboutir aux articles de Graffici.ca.

- Pour ce qui est des articles, c'est une information de proximité deux jeunes perdus en forêt - qui a battu tous les records, soit le quart des pages vues.

- Comparant la performance des 31 jours de mai 2012 par rapport aux 31 jours d'octobre 2011, on découvre une croissance de $61,18 \%$ des visites et de 33,96\% des pages vues.

II n'est pas nécessaire, pour le propos de ce texte, d'aller plus loin dans le détail des informations et croisements de données que Google Analytics permet. II suffit en effet de noter que chacune de ces propositions générales peut donner lieu à des interprétations pour les décisions stratégiques de l'entreprise afin d'augmenter la fréquentation: faire jouer les statistiques de fréquentation et de croissance auprès des annonceurs, mieux utiliser Facebook pour le recrutement, mieux positionner et faire des activités de promotion de la marque Graffici.ca pour que davantage d'internautes soient attirés sur la page d'accueil, etc. II importe cependant de souligner ici que les données plus détaillées de fréquentation par thèmes et mots-clés étaient largement inaccessibles avant Internet, à savoir la fréquentation de chaque page, de chaque thème, de chaque article, de chaque photo, de chaque chroniqueurblogueur.

Aujourd'hui, il est possible de réaliser un classement de chaque contenu en terme de popularité et de suggérer des mises en évidence potentiellement plus efficaces, l'usage de mots plus populaires - sur l'Internet, les mots-clés des titres sont des outils de référencement dans les moteurs de recherche - des choix de couverture plus attractifs, etc. II faut garder en mémoire aussi les possibilités nombreuses de collecte de données et d'analyses plus complexes qu'offrent les services gratuits et payants de Google, ou d'autres firmes, au-delà 


\section{François DEMERS}

des données de base qui ont été examinées ici. L'exercice donne cependant un aperçu du monde qui s'ouvre pour le marketing médiatique et pour la gouverne du journalisme à l'intérieur des médias qui continueront à en faire leur locomotive, leur image de marque, leur offre principale ou un simple produit d'accompagnement.

\section{Dévoilement vs utopie}

Comme le montrent ces trois cas, les nécessités commerciales contraignent les acteurs à pénétrer plus avant dans la course aux profilages et même à donner un coup d'accélérateur en utilisant le potentiel de cueillette et d'analyse des données que promettent les grands opérateurs de l'Internet. Pour les salles de rédaction journalistique, cela suppose un mode étendu et systématique de dissémination interne des informations ainsi recueillies. Car des moyens techniques et des pratiques se mettent en place, qui permettent d'évaluer les productions individuelles ou collectives - car la majorité des productions journalistiques continuent de nécessiter le travail en équipe - à l'aune de leur capacité à attirer, à retenir et à fidéliser un bloc significatif de consommateurs.

Dans le contexte d'hyperconcurrence entre les entreprises du secteur des médias, entre les messages offerts en public, entre les propositions d'activités pour l'occupation du budget-temps des lecteurs, cybernautes et consommateurs, les pressions sont énormes pour que les journalistes, comme autres employés des entreprises se plient aux nécessités du commerce et alignent leur production sur celle qui paraît pouvoir assurer le financement de l'entreprise qui les emploie. Chaque journaliste doit devenir attentif et sensible à ces données. Car ont sauté les points d'ancrage qui permettaient auparavant à la majorité des membres de la confrérie de se considérer comme des professionnels. Au point que la représentation traditionnelle du journalisme comme un service à un public prédéfini dans l'abstrait, en tant qu'ensemble de citoyens à former et à informer, se réoriente vers la simple satisfaction du consommateur.

Le contexte médiatique actuel est habité par une culture qui promeut et célèbre la réussite individuelle et l'entrepreneuriat, reléguant le lieu du bien commun aux seules fonctions régaliennes de l'État - la police, l'armée et la perception des impôts pour entretenir les deux premières fonctions. Les journalistes apparaissent conséquemment de plus en plus comme des serviteurs d'intérêts particuliers et non comme des promoteurs du bien commun. Comme si leur véritable jeu avait été " dévoilé » au sens de Pierre Bourdieu en révélant la base économique de leurs comportements. Le regard collectif, cynique, devient graduellement leur à propos d'eux-mêmes: serviteurs 
Le défi de la construction d'un public pour le journalisme au temps de Google Analytics

consentants sinon enthousiastes de leur employeur. II les disqualifie pour le rôle noble de soutien à la démocratie qu'ils affirmaient que la société leur avait confié et qu'ils prétendaient jouer envers et contre tous (Demers 1997).

Dès lors, l'avenir est déjà à des mots d'ordre comme celui que dénonçait récemment la journaliste bien connue Josée Blanchette ${ }^{18}:$ « Je vous parlais des médias un peu plus haut, où j'ai vu s'insinuer lentement le culte du "sujet payant", surtout depuis que le nombre de clics, de retweets et de "j'aime" nous définit comme "fournisseurs de contenu". Comme le dit une de mes boss(e) : "Quand je veux être certaine de ne pas être lue, j'écris sur la pauvreté ou les Autochtones." J'ajouterais: les chômeurs, une variable statistique de la pauvreté. 》 (Blanchette, 2012)

Sur le terrain de l'information de qualité, prédit-on, ne resteront bientôt que quelques médias qui continueront à placer, au centre de leur image de marque ou comme produit principal, un contenu destiné au segment de consommateurs qui se veulent citoyens éclairés ou cherchant à l'être. Peut-être que ces médias de niche, par le jeu tautologique du marketing, vont contribuer au maintien des inégalités culturelles entre groupes et même au développement des inégalités au profit de leurs publics, plutôt qu'à maintenir vivant l'objectif d'amélioration de la démocratie. Comme aux États-Unis, dont l'écologie médiatique est depuis longtemps dominée par le marketing (Ravault, 1983).

Peut-être aussi qu'en sens contraire, le bien commun est-il en voie de relégitimation, en partant du cœur d'Internet, autour du thème des logiciels libres et des creative commons, étant donné le fil historique dont parle Armand Mattelart : "Dans l'histoire du mouvement social, un fil rouge est apparent, qui court entre l'idée de biens publics communs et l'utopie du service public comme appropriation collective des domaines d'utilité de tous [...]. » (Mattelart, Sénécal, $2011: 276)$.

18 Chroniqueuse au Devoir et blogueuse au magazine Châtelaine. http://fr.wikipedia.org/wiki/Jos\%C3\%A9e Blanchette, consultée le 6 août 2012 


\section{François DEMERS}

\section{Bibliographie}

ANDERSON B., 1996, L'imaginaire national. Réflexions sur l'origine et l'essor du nationalisme, Paris, La Découverte.

BERNIER M.-F., 2008, Journalistes au pays de la convergence. Sérénité, malaise et détresse dans la profession, Québec, Les Presses de l'Université Laval.

BOURDIEU P., 1994, Raisons pratiques - Sur la théorie de l'action, Paris, Seuil, Essais.

BRIN C., J. CHARRON et J. DE BONVILLE, 2004, Nature et transformation du journalisme. Théorie et recherches empiriques, Québec, Les Presses de l'Université Laval, 454 pages.

COSTEY P., 2009, “L'illusio chez Pierre Bourdieu. Les (més)usages d'une notion et son application au cas des universitaires ", Tracés. Revue de sciences humaines, Lyon, ENS Éditions, <http://traces.revues.org/2133> Dernière consultation: 19 août 2012.

DEMERS F., 2003, “"Fenêtres d'opportunité" et émergence de priorités politiques Le cas du glissement de la recherche des effets des médias vers l'étude de la réception », dans J. CRËTE (dir.). Hommage à Vincent Lemieux. La science politique au Québec. Le dernier des maîtres fondateurs, Québec, Les Presses de l'Université Laval, pp. 434-452.

DEMERS F., 1997, « Journalisme : à propos d'une "demande éthique" d'une ampleur anormale », Ethica, Groupe de recherche ETHOS, Université du Québec à Rimouski. Vol. 9, $\mathrm{n}^{\circ}$ 2, tome 11, Septembre, pp. 297-322.

DEMERS F., 1989, "Journalistic ethics: The rise of the "good employee's model": a threat for professionalism ? ", The Canadian Journal of Communication, vol. 14, $\mathrm{n}^{\circ}$ 2, May 1989, pp. 15-27.

GAGNÉ J.-P., 1980, «L'information, un produit comme les autres », dans J. DE BONVILLE, G. LESAGE et F. SAUVAGEAU (dir.), Dans les coulisses de l'information - Les journalistes, Montréal, Éditions Québec / Amérique, pp. 73-97.

GIROUX D., 2012, "Les médias en quelques statistiques », dans Institut du Nouveau Monde, L'état du Québec 2012. Montréal, Les Éditions du Boréal, pp. 356-362. 
Le défi de la construction d'un public pour le journalisme au temps de Google Analytics

HALL S., 2007, « La question multiculturelle », dans S. HALL, 2007, Identités et cultures - Politiques des cultural studies, Éditions Amsterdam, Paris, pp. 289-327.

HABERMAS J., 1968, L'espace public. Archéologie de la publicité comme dimension constitutive de la société bourgeoise. Paris, Payot, réed.

MATTELART A., M. SÉNÉCAL, (2011). Pour un regard-monde. Entretiens avec Michel Sénécal, Montréal, Les Presses de l'Université de Montréal.

MIÈGE B., 1995, «L'espace public : perpétué, élargi et fragmenté », dans I. PAILLART (dir.), L'espace public et l'emprise de la communication, éditions Ellug, Grenoble, pp. 163 à 173 .

MERRILL JC., 1968, The Elite Press - Great Newspapers of the World. New York, Toronto, London : Pitman Publishing Corporation, 336 pages.

MUHLMANN G., 2004, Du journalisme en démocratie, Paris : Éditions Payot \& Rivages, 347 pages.

PRONOVOST G., M.-È. BEAUMIER, avec la collaboration de C. LEGAULT, 2012, «Typologie des représentations du public chez les acteurs du multimédia », dans C. MARTIN, M. DE LA DURANTAYE, J. LEMIEUX et J. LUCKERHOFF (dir.). Enjeux des industries culturelles au Québec - Identité, mondialisation, convergence, pp. 241-251.

RAVAULT R.-J., 1983, «Exhibitionnisme naïf ou stratégies de la communication ", dans Communication, vol. 6, $n^{\circ} 1$, Québec, Département d'information et de communication, Université Laval, pp. 65-80.

REBILLARD F., 2011, "L'étude des médias est-elle soluble dans l'informatique et la physique? À propos du recours aux digital methods dans l'analyse de l'information en ligne ", Questions de communication, $\mathrm{n}^{0} 20$, Notes de recherche, pp. 353-376.

RIEFFEL R., 2005, Sociologie des Médias, Paris, Ellipses, $2^{\mathrm{e}}$ édition, pp. 163185 (II - Le public).

RINGOOT R. et J.-M. UTARD, 2005, « Genres journalistiques et "dispersion" du journalisme ", dans RINGOOT, R. et J.-M. UTARD (dir.), Le journalisme en invention. Nouvelles pratiques, nouveaux acteurs, Rennes, Presses Universitaires de Rennes, pp. 21-47. 


\section{François DEMERS}

THOMPSON JB., 1995, "The Tranformation of Visibility », dans The Media and Modernity - A Social Theory of the Media, London, Polity Press, pp. 119148.

TÉTU J.-F., 2008, «Transformations et dispersion du journalisme, en France », dans D. AUGEY, F. DEMERS et J.-F. Tétu (dir.). Figures du journalisme Brésil, Bretagne, France, La Réunion, Mexique, Québec. Québec, Les Presses de l'Université Laval, $1^{\text {er }}$ trimestre, pp. 19-45

\section{Documents divers}

AGENCE FRANCE-PRESSE, 2012, "YouTube, de plus en plus un média d'information, selon une étude ", Le Devoir, 17 juillet, page B7. Voir aussi : http://www.journalism.org/sites/journalism.org/files/YouTube\%20\&\%20the\%2 0News\%20-\%20A\%20PEJ\%20Report\%20-\%20FINAL 0.pdf, consulté le 3008-2012

ALLARD M., 2012, "Voir Québec perd son rédacteur en chef 》, Le Soleil, 20 juin, page 47.

BAILLARGEON S., 2012a, "C'est triste à Voir. Hour et deux hebdomadaires culturels ferment. ", Le Devoir, 4 mai, p. B2.

BAILLARGEON S., 2012 b, "Débranchez-vous! Rogers ferme le portail Branchez-vous et sept autre sites », Le Devoir, 2 mai, p. B8.

BAILLARGEON S., 2012c, «Publications : le lectorat reste stable, Mais la chute des revenus publicitaires menace les journaux », Le Devoir, 3 / 4 avril, p. B9.

BAILLARGEON S., 2012d, " Le cube Oxo », Le Devoir, 19 mars, p. B7.

BAILLARGEON S., 2012e, " Médias - Magazines. Le grand recul se poursuit », Le Devoir, 14 mars.

BAILLARGEON S., 2012f, "Citizen Ariana - Le Huffington Post Québec est né ", Le Devoir, 9 février, p. A1 et A10.

BAILLARGEON S., 2012g, "Trente d'un coup - Le Voir diffuse une masse de nouveaux blogues ", Le Devoir, 12 janvier, p. B8.

BELOT L., 2011, « Twitter, le révélateur », Le Devoir, 17 janvier, page B7 (Texte reproduit du quotidien Le Monde).

BLANCHETTE J., 2012, "Mon nom est personne - Perdre son job dans un monde de MBA », Le Devoir, 30 mars, p. B12 (Zeitgeist). 
Le défi de la construction d'un public pour le journalisme au temps de Google Analytics

CAUCHON P., 2010, «La relation entre les magazines et le Web sous un nouvel éclairage ", Le Devoir, 8 mars, p. B7.

DEMERS F., 2012, "Le portail Web Graffici.ca un "laboratoire vivant" de développement territorial ", communication présentée le 8 mai dans le cadre du colloque Innovation sociale et coopération: une nouvelle génération de recherche organisé par le Centre de recherche sur les innovations sociales (CRISES), Montréal, ACFAS 2012.

GUAY J.-H., 2009, "Bilan de la décennie: la citoyenneté québécoise recomposée ", Le Devoir, 31 décembre, p. C6.

MOFFETTE D., 2009, "Réinventer un espace public hors de la nation », Le Devoir, 30 juin, p. A7.

ROBITAILLE A., 2009, «Fin des journaux, fin des nations ? », Le Devoir, 27/28 juin, pp. A1 et $A 10$.

SAULNIER A., 2012, "Les défis du journalisme québécois », Le Devoir, 6 juillet, p. A9.

STATE OF THE MEDIA, 2012. http://stateofthemedia.org/2012/overview-4/

TURCOTTE C., 2012, "Les entrevues HEC Montréal - Et demain, le neuromarketing ! - HEC Montréal dispose de nouveaux outils pour mieux cerner les consommateurs ", Le Devoir, 11 avril, pp. B1 et B4. 